$\operatorname{IPPP} / 05 / 54$

\title{
Neutron-Electron EDM Correlations in Supersymmetry and Prospects for EDM Searches
}

\author{
Steven A. Abel \\ IPPP, University of Durham, DH1 3LE Durham, UK \\ Oleg Lebedev \\ Deutsches Elektronen-Synchrotron DESY, 22603 Hamburg, Germany
}

1st November 2021

\begin{abstract}
Motivated by recent progress in experimental techniques of electric dipole moment (EDM) measurements, we study correlations between the neutron and electron EDMs in common supersymmetric models. These include minimal supergravity (mSUGRA) with small CP phases, mSUGRA with a heavy SUSY spectrum, the decoupling scenario and split SUSY. In most cases, the electron and neutron EDMs are found to be observable in the next round of EDM experiments. They exhibit certain correlation patterns. For example, if $d_{n} \sim 10^{-27} e \mathrm{~cm}$ is found, $d_{e}$ is predicted to lie in the range $10^{-28}-10^{-29} \mathrm{e} \mathrm{cm}$.
\end{abstract}




\section{Introduction}

The current EDM limits $[1]-[3]^{1}$

$$
\begin{aligned}
& \left|d_{n}\right|<6 \times 10^{-26} e \mathrm{~cm}, \\
& \left|d_{e}\right|<2 \times 10^{-27} e \mathrm{~cm}, \\
& \left|d_{\mathrm{Hg}}\right|<2 \times 10^{-28} e \mathrm{~cm}
\end{aligned}
$$

impose severe constraints on CP violating physics beyond the Standard Model and, in particular, supersymmetry. Supersymmetric models accommodating the above bounds usually predict EDMs not far from the current limits which makes the next round of EDM experiments with the sensitivity $d_{n} \sim 10^{-28} e \mathrm{~cm}, d_{e} \sim 10^{-30} e \mathrm{~cm}$ [4] - [8] particularly interesting. Other EDM experiments such as measurements of the deuteron and muon EDMs [9] would provide important complementary information on $\mathrm{CP}$ violation in supersymmetry.

In this work, we address the questions "What are the EDM expectations in SUSY models ?" and "How can one distinguish different sources of EDMs ?". It is well known that generic SUSY models predict too large EDMs which constitutes the SUSY CP problem [10]. The problem is resolved in certain classes of supersymmetric models, of which we choose four representative types. These include SUSY models with

- small CP phases

- heavy spectrum

- decoupling

- split SUSY

To determine the source of EDMs, we study correlations between the neutron and electron electric dipole moments. In particular, if the neutron EDM is due to the QCD $\theta$-term [11] - [13], one expects very small leptonic EDMs. On the other hand, in supersymmetry both the hadronic and leptonic EDMs are enhanced and there exist certain correlation patterns. Determination of such patterns is the main subject of this work.

The relevant low energy Lagrangian describing interactions of electrons, quarks, gluons and photons is given by

$$
\mathcal{L}=\theta \frac{\alpha_{s}}{8 \pi} G \tilde{G}+\frac{1}{3} w f^{a b c} G^{a} \widetilde{G}^{b} G^{c}-\frac{i}{2} d_{f} \bar{f}(F \sigma) \gamma_{5} f-\frac{i}{2} g_{s} \widetilde{d}_{q} \bar{q}(G \sigma) \gamma_{5} q,
$$

where $w, d_{f}, \tilde{d}_{q}$ are the Weinberg operator [14] coefficient, the fermion EDM, and the quark chromo-EDM (CEDM), respectively. Here $F$ and $G$ represent the photon and gluon field strengths, respectively. The electron EDM is given by $d_{e}$, whereas

\footnotetext{
${ }^{1}$ The EDM collaboration has recently announced an improved (preliminary) neutron EDM bound $\left|d_{n}\right| \lesssim 3 \times 10^{-26}$ e cm [4].
} 
the neutron EDM is a model-dependent function of $\theta, w, d_{q}$ and $\tilde{d}_{q}$. We will use the naive dimensional analysis (NDA) approach $[15],{ }^{2}$

$$
d_{n}^{\mathrm{NDA}} \sim 2 d_{d}-0.5 d_{u}+e\left(0.4 \tilde{d}_{d}-0.1 \tilde{d}_{u}\right)+0.3 \mathrm{GeV} \times e w
$$

where we have set $\theta=0$. Here the Wilson coefficients $d_{q}, \tilde{d}_{q}, w$ are evaluated at the electroweak scale. The chromo-EDM and the Weinberg operator contributions involve considerable uncertainties. Furthermore, the result is sensitive to the quark masses which we choose as $m_{u}\left(M_{Z}\right)=2 \mathrm{MeV}$ and $m_{d}\left(M_{Z}\right)=4 \mathrm{MeV}$. The approach based on QCD sum rules gives a somewhat similar result [17],

$$
d_{n}^{\mathrm{SR}} \simeq 2 d_{d}-0.5 d_{u}+e\left(\tilde{d}_{d}+0.5 \tilde{d}_{u}\right)+0.1 \mathrm{GeV} \times e w .
$$

In the Weinberg operator contribution, we have used the (model-dependent) renormalization factor of Ref. [15]. In practice, the two approaches usually agree within a factor of 2 (unless $w$ dominates) which suffices for our purposes. We note that there are also neutron models which include the strange quark contribution [18]. This effect is difficult to estimate and involves large uncertainties. We defer a study of such models until a subsequent publication.

\section{$2 \quad$ EDMs in the Standard Model}

We start by considering EDMs induced by the QCD $\theta$-term. This is the primary source of hadronic EDMs in the Standard Model [11] - [13], [19]. In particular [13],

$$
d_{n} \simeq 3 \times 10^{-16} \theta \text { e cm }
$$

with about $50 \%$ uncertainty. On the other hand, the electroweak contributions are very small, $d_{n} \sim 10^{-32} e \mathrm{~cm}[20]$. To satisfy the experimental bound (11), the $\theta$ parameter has to be tiny, $\leq \mathcal{O}\left(10^{-10}\right)$. Such a small value can hardly be explained by the Standard Model, which constitutes the "strong CP problem". The most popular solution to this problem in extensions of the SM invokes an anomalous Peccei-Quinn symmetry [21], which sets $\theta$ to zero. However, this symmetry is expected to be broken by higher dimensional operators generated at the Planck scale [22], such that the resulting $\theta$ is finite but small. There are also alternative solutions to the strong CP problem which employ other symmetries [23]. In these models, a finite $\theta$ can be induced by radiative corrections. In either case, a small neutron EDM is expected but it is difficult to make any quantitative prediction.

The $\theta$-term also induces nuclear EDMs. In particular, it generates EDMs of the deuteron and the mercury atom. The former receives contributions from both the

\footnotetext{
${ }^{2}$ The QCD correction factor for the $d_{u, d}$ contributions has recently been recalculated [16] and found to be a factor of 2 or so smaller than that in [15]. This leads to somewhat smaller estimates for $d_{n}$.
} 
constituent nucleons and nucleon interactions, and can be evaluated via QCD sum rules with about 50\% uncertainty [24]. An EDM of the mercury atom is induced by the Schiff moment which appears due to $\mathrm{CP}$ violating isoscalar and isovector pionnucleon couplings. Despite recent progress in evaluating these contributions [25], there is still an order of magnitude uncertainty in $d_{\mathrm{Hg}}(\theta)$, whereas $d_{\mathrm{Hg}}$ induced by the quark (colour-) EDM contributions is understood much better. The result is

$$
\begin{aligned}
& d_{D} \simeq-1 \times 10^{-16} \theta \text { e } \mathrm{cm}, \\
& \left|d_{\mathrm{Hg}}\right| \sim \mathcal{O}\left(10^{-18}-10^{-19}\right) \theta \text { e } \mathrm{cm} .
\end{aligned}
$$

The above formulae together with suppressed leptonic EDMs provide a correlation pattern for the $\theta$-induced electric dipole moments.

It is important to remember that $d_{e}$ is not measured directly, but instead is derived from atomic EDMs. In particular, the current EEDM bound is due to the thallium atom EDM measurement [2] and the relation $d_{\mathrm{Tl}} \simeq-585 d_{e}$. If the $\theta$-term is non-zero, this relation is altered. Although no reliable calculation of $d_{\mathrm{Tl}}(\theta)$ is available, rough estimates [26] of the valence proton EDM contribution give $d_{\mathrm{Tl}} \sim 10^{-20} \theta$ e $\mathrm{cm}$. On the other hand, the SM electroweak interactions (including Majorana neutrinos) usually induce $d_{e}$ of order $10^{-38} e \mathrm{~cm}$ [27]. Thus, in the Standard Model with $\theta>10^{-15}, d_{\mathrm{Tl}}$ is dominated by the $\theta$-background and is less sensitive to $d_{e}$. This illustrates that the effects of the $\theta$-term can be important for atomic systems and should be taken into account.

\section{$3 \quad$ EDMs in supersymmetry}

In supersymmetric models, there are additional sources of $\mathrm{CP}$ violation associated with complex phases in the SUSY breaking F-terms and, in addition, flavour misalignment between the Yukawa matrices and the soft breaking terms [28]. EDMs are generated already at the one loop level and typically exceed the experimental bounds by orders of magnitude [10]. This constitutes the SUSY CP problem. The problem is alleviated in certain classes of supersymmetric models. These include models with small SUSY CP phases, those with a heavy SUSY spectrum, the decoupling scenario and split SUSY. In what follows, we study neutron-electron EDM correlations in these types of models.

We note that there are also other possibilities for EDM suppression. For instance, $\mathrm{CP}$ violation may have flavour off-diagonal nature due to some symmetry [29], [30]. We defer a study of this option until a subsequent publication. Fi-

nally, EDMs may be suppressed due to accidental cancellations among independent terms [31]. This option is however disfavoured by the mercury EDM constraint [32] $-[35]$.

In our numerical analysis, we choose two representative values of $\tan \beta, 5$ and 35, and analyze the NEDM-EEDM correlations separately for SUSY CP violation 


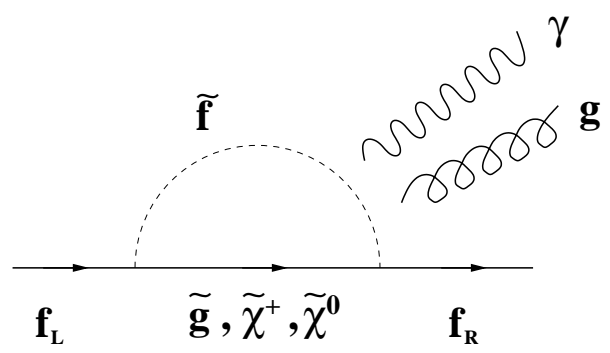

Figure 1: One loop SUSY contributions to fermion (C)EDMs.

induced by the phase of the $\mu$-term, $\phi_{\mu}$, and CP violation due to the phase of the trilinear A-terms, $\phi_{A}$. We scan over the parameter space of a given model and present our results as scatter plots $d_{e}$ vs $d_{n}$.

\subsection{Small CP phases}

First, we study the minimal SUGRA model (mSUGRA) with small $\left(10^{-2}\right) \mathrm{CP}$ phases. The smallness of the physical phases may be due to approximate alignment between the phases of the soft terms as occurs in the dilaton-domination scenario [36] with the Giudice-Masiero mechanism for the $\mu$-term, or due to some approximate symmetry [37].

The model is defined in terms of the following GUT scale parameters,

$$
m_{0}, m_{1 / 2},|A|, \tan \beta
$$

and $\mathrm{CP}$-phases $\phi_{A}, \phi_{\mu}$. Here $m_{0}$ is the universal scalar mass, $m_{1 / 2}$ is the universal gaugino mass and $A$ is the trilinear parameter. The Higgs potential parameters $|\mu|$ and $|B \mu|$ are found by imposing radiative electroweak symmetry breaking. We assume that the $\mathrm{CP}$ phases associated with the gaugino mass and the $B \mu$-term have been rotated away by appropriate $U(1)_{\mathrm{R}}$ and $U(1)_{\mathrm{PQ}}$ transformations. In this case, the physical CP phases are parametrized by $\phi_{A}$ and $\phi_{\mu}$.

The (C)EDMs are dominated by one-loop diagrams involving gluinos, charginos and neutralinos, Fig 1.

$$
\begin{aligned}
& d_{q}=d_{q}^{\tilde{g}}+d_{q}^{\tilde{\chi}^{+}}+d_{q}^{\tilde{\chi}^{0}} \\
& \tilde{d}_{q}=\tilde{d}_{q}^{\tilde{g}}+\tilde{d}_{q}^{\tilde{\chi}^{+}}+\tilde{d}_{q}^{\tilde{\chi}^{0}} \\
& d_{e}=d_{e}^{\tilde{\chi}^{+}}+d_{e}^{\tilde{\chi}^{0}} .
\end{aligned}
$$

The two loop contributions are considerably smaller. The relevant formulae can be found, for example, in Ibrahim and Nath, Ref. [31].

To get a feeling for the size of the EDMs, let us consider a simple approximation $\phi_{\mu} \sim \phi_{A} \equiv \phi \ll 1, \tan \beta \sim 3$ and assume a single mass scale $M$ for the SUSY 
parameters (at the electroweak scale). Then,

$$
\begin{aligned}
& d_{n} \sim\left(\frac{300 \mathrm{GeV}}{M}\right)^{2} \sin \phi \times 10^{-24} e \mathrm{~cm}, \\
& d_{e} \sim\left(\frac{300 \mathrm{GeV}}{M}\right)^{2} \sin \phi \times 10^{-25} e \mathrm{~cm} .
\end{aligned}
$$

Both $d_{n}$ and $d_{e}$ grow linearly with $\tan \beta$. Clearly, for the SUSY spectrum with electroweak masses, the CP phase has to be of order $10^{-2}$. We see that the neutron and electron EDMs differ by about an order of magnitude, however no prediction of their magnitudes can be made since the result depends on how small the phase is. If no $d_{e}$ at the level $10^{-30} e \mathrm{~cm}$ is found, the CP phase will have to be smaller than $10^{-5}$ which appears highly unnatural. It would be rather difficult to engineer a robust mechanism which would force the phases in the Lagrangian to align with such an extraordinary precision. Thus, one may argue that $d_{e}$ and $d_{n}$ in this scenario should not be far below the current experimental limits.

In the same simple approximation, the mercury atom EDM is given by $d_{\mathrm{Hg}} \sim$ $(300 \mathrm{GeV} / M)^{2} \sin \phi \times 10^{-26} e \mathrm{~cm}$. Here $d_{\mathrm{Hg}}$ is dominated by the quark CEDMs [32] and we have included an extra factor $\sim 1 / 4$ due to a recent reevaluation of the nuclear/atomic matrix elements. The deuteron EDM is expected to be similar to the neutron EDM, $d_{D} \sim d_{n}$.

Let now turn to our numerical results presented in Figs. 617, In these plots, we vary $m_{0}, m_{1 / 2},|A|$ randomly in the range $200 \mathrm{GeV}-1 \mathrm{TeV}$ and the phases $\phi_{A}, \phi_{\mu}$ in the range $[-\pi / 500, \pi / 500]$. In the left plot, $\phi_{A}$ is set to zero and, in the right plot, $\phi_{\mu}=0$. For non-zero $\phi_{\mu}$, there is a clear linear $d_{e}-d_{n}$ correlation and $d_{e}$ is about an order of magnitude below $d_{n}$, as expected. $d_{e}$ is dominated by the chargino diagram, whereas $d_{n}$ receives comparable contributions from the charginos and gluinos. At higher $\tan \beta$, the EDMs increase linearly. For $\phi_{A} \neq 0$, the correlation

is less pronounced. For a given $d_{n}$, the spread of $d_{e}$ values is about two orders of magnitude. This is because $d_{n}$ is dominated by the gluino diagrams, whereas $d_{e}$ is dominated by the neutralino contributions. The former are relatively insensitive to $m_{0}$ since the squark masses are dominated by the gluino RG contributions, whereas the latter are sensitive to $m_{0}$ through both the slepton masses and the $\mu$-parameter. Thus, fixing $d_{e}$ does not determine $d_{n}$ accurately. Note that EDMs induced by $\phi_{A}$ do not receive $\tan \beta$-enhancement.

\subsection{Heavy SUSY spectrum}

The SUSY contributions to EDMs are suppressed if the entire SUSY spectrum is in the TeV range (Eq.(8) ). Such a possibility is motivated by the strong bound on the Higgs mass which requires the stop mass to be of order $1 \mathrm{TeV}$. If all SUSY masses are controlled by the same scale, the spectrum is heavy. This senario can be motivated in various ways, see e.g. Ref. [38]. 

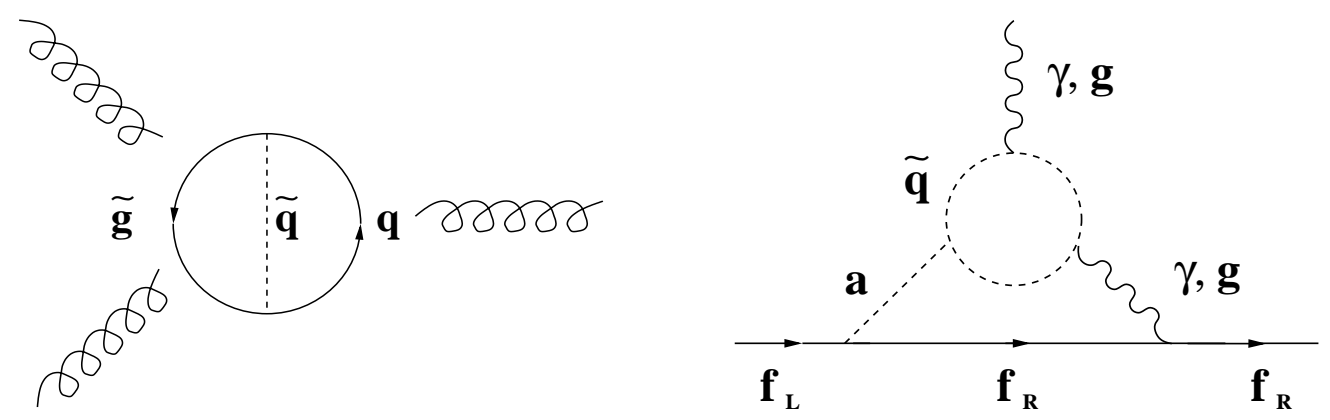

Figure 2: 2 loop EDM contributions. The diagram on the left induces the Weinberg operator. On the right, is an example of the Barr-Zee type diagram ( $a$ is a pseudoscalar Higgs).

In this class of models, the EDMs are usually dominated by the one loop diagrams of Fig [1 and the analysis is very similar to that presented in the previous subsection. We note that if the $d_{e}$ experiments with the $10^{-30} e \mathrm{~cm}$ sensitivity yield a null result, it would imply that the scale of SUSY masses is $100 \mathrm{TeV}$ (Eq.(8)). Models with such a high SUSY breaking scale are disfavoured by the gauge coupling unification and naturalness considerations. Thus, again one expects a non-zero result in the next round of EDM experiments.

In our numerical analysis, we study the mSUGRA model (Eq.(6) ) with $m_{0}, m_{1 / 2},|A|$ in the range $2 \mathrm{TeV}-10 \mathrm{TeV}$ and $\phi_{A}, \phi_{\mu}$ in the range $[-\pi, \pi]$. Our results are shown in Figs. 8.9. The $d_{n}-d_{e}$ correlation patterns are very similar to those in the small phase scenario, namely there is a well defined correlation in the $\phi_{\mu} \neq 0$ case, whereas for $\phi_{A} \neq 0$ it is far less pronounced.

\subsection{Decoupling}

The largest contributions to EDMs come from sfermions of the first two generations. If these are very heavy, $>10 \mathrm{TeV}$, the most dangerous contributions are suppressed [39]. The third generation is required to be light by naturalness and contributes to EDMs at the 2 loop level. This decoupling scenario can be realized, for instance, in certain types of GUT models where the hierarchy between the first two and third generation masses appears due to RG running [40].

The neutron EDM is dominated by the stop and sbottom contributions to the Weinberg operator [41], whereas the electron EDM is due to the Barr-Zee [42] type 2 loop diagrams [43], Fig[2] This class of diagrams also includes graphs with internal charginos and $h, H, A$ Higgs bosons [44]. Thus,

$$
\begin{aligned}
& d_{n} \simeq d_{n}(w), \\
& d_{e}=d_{e}^{\operatorname{Barr}-\operatorname{Zee}\left(\tilde{f}, \tilde{\chi}^{+}, \tilde{\chi}^{0}\right)} .
\end{aligned}
$$

We note that the Barr-Zee type diagrams also contribute to the neutron EDM, 

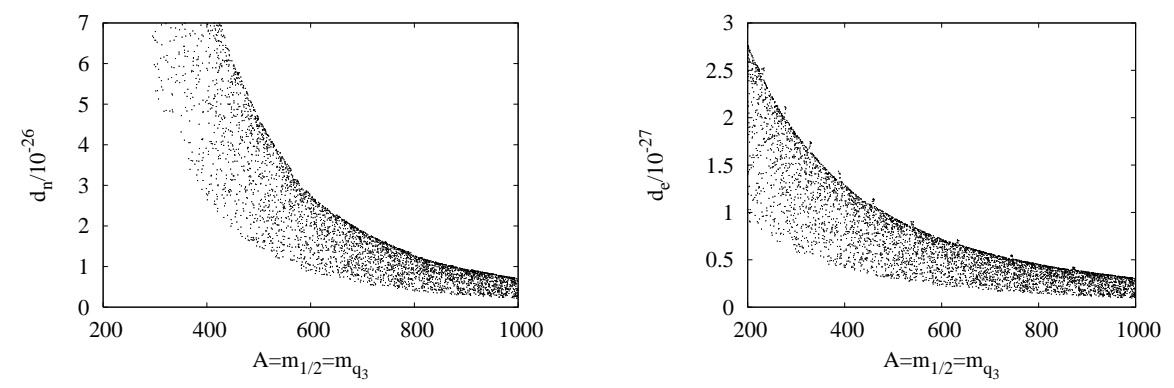

Figure 3: $d_{n}^{\mathrm{NDA}}$ (left) and $d_{e}$ (right) in the decoupling scenario, $\phi_{\mu} \in[\pi / 10, \pi / 2], \tan \beta=$ 3. The GUT scale parameters $A=m_{1 / 2}=m_{\tilde{q}_{3}}$ are in $\mathrm{GeV}$ and the EDMs are in $e \mathrm{~cm}$. The QCD sum rules model gives $d_{n}^{\mathrm{SR}}$ a factor of a few smaller.

but these are suppressed compared to the Weinberg operator [34]. The correlation between $d_{e}$ and $d_{n}$ is rather subtle since $d_{n}$ depends on the gluino and the third generation squark masses, whereas $d_{e}$ depends on the latter as well as the chargino and Higgs masses. An order of magnitude estimate of the resulting EDMs can be obtained by setting all SUSY masses (apart from the first two generation sfermions) to be $M \gg M_{Z}$ and the CP phases to be given by a single quantity $\phi$. Then, for moderate $\tan \beta[41]-[44]$,

$$
\begin{aligned}
& d_{n} \sim\left(\frac{300 \mathrm{GeV}}{M}\right)^{2} \tan \beta \sin \phi \times 10^{-25} e \mathrm{~cm}, \\
& d_{e} \sim\left(\frac{300 \mathrm{GeV}}{M}\right)^{2} \tan \beta \sin \phi \times 10^{-27} e \mathrm{~cm} .
\end{aligned}
$$

Here $d_{n}$ and $d_{e}$ differ by almost two orders of magnitude, whereas in other scenarios this hierarchy is usually a factor of ten.

In this approximation, the mercury EDM is less sensitive to the CP phase $\phi$. This is because the Weinberg operator contribution to $d_{\mathrm{Hg}}$ is negligible and the EDM is generated by the subleading quark CEDMs. When $M$ or $\phi$ in Eq. (10) is adjusted to satisfy the current neutron EDM bound, the resulting $d_{\mathrm{Hg}}$ is $\leq \mathcal{O}\left(10^{-29}\right) e$ $\mathrm{cm}$, which is likely to be below the reach of the mercury EDM experiments. The deuteron EDM, however, is again found to be similar to $d_{n}$.

In our numerical analysis, we use the following GUT input parameters

$$
m_{3}, m_{1 / 2},|A|, \tan \beta
$$

and CP phases $\phi_{A}, \phi_{\mu}$. Here $m_{3}$ is the mass parameter for all the scalars apart from those for the first two sfermion generations, which are assumed to be decoupled. As in the mSUGRA case, $|\mu|$ and $|B \mu|$ are determined by radiative electroweak symmetry breaking. We vary $m_{3}, m_{1 / 2},|A|$ in the range $200 \mathrm{GeV}-1 \mathrm{TeV}$ and $\phi_{A}$, $\phi_{\mu}$ in the range $[-\pi, \pi]$. Fig 3 shows typical ranges of the $d_{n}$ and $d_{e}$ values for order one CP phases ${ }^{3}$. Clearly, $d_{n} \sim 10^{-26} e \mathrm{~cm}$ and $d_{e} \sim 10^{-27}-10^{-28} e \mathrm{~cm}$ are expected

\footnotetext{
${ }^{3} d_{n}$ and $d_{e}$ are dominated by contributions sensitive to $\phi_{\mu}$.
} 
in this scenario. Smaller EDMs would imply that either the CP phases are small or that the SUSY spectrum is heavy, including the third generation sfermions.

The corresponding correlations are presented in Figs. 10 11] For $\phi_{\mu} \neq 0, d_{e}$ and $d_{n}$ can differ by two or one order of magnitude, depending on the balance between the Weinberg operator and the chargino Barr-Zee contributions. At larger $\tan \beta$, the situation remains the same except the EDMs increase proportionally. Note that since we scan over $\mathrm{CP}$ phases in the region $[-\pi, \pi]$, we include the possibility that the $\mathrm{CP}$ phases are small such that smaller values of the EDMs compared to those in Fig 3 are allowed. For $\phi_{A} \neq 0$, the hierarchy between $d_{n}$ and $d_{e}$ increases to three or four orders of magnitude. This is because $d_{n}$ is dominated by the stop contribution to the Weinberg operator, whereas the leading chargino Barr-Zee contribution to $d_{e}$ is now absent and $d_{e}$ is due to the stop Barr-Zee diagram. At larger $\tan \beta$, this hierarchy reduces since unlike the Weinberg operator, Barr-Zee contributions receive $\tan \beta$-enhancement.

\subsection{Split SUSY}

This is an extreme version of the decoupling scenario in which the third generation sfermions are decoupled as well and naturalness is abandoned [45], [46]. From the EDM perspective, it provides an interesting framework in which the neutron and electron EDMs are generated by the same type of diagrams and thus are highly correlated.

The EDMs are induced by a version of the Barr-Zee type diagram, Fig [2] with the sfermion loop replaced by the chargino loop [44]. Since all the Higgses except for the SM-like $h$ are assumed to be heavy, the chargino loop is attached to the fermion line through $h$ and a photon [46], or $W^{+}$and $W^{-}[47] .{ }^{4}$ The relevant formulae can be found in Refs. [44], [47]. Clearly, the contributing diagrams are the same for the electron and the neutron such that the EEDM and NEDM are strongly correlated. We have therefore,

$$
\begin{aligned}
& d_{q}=d_{q}^{\operatorname{Barr}-\operatorname{Zee}\left(\tilde{\chi}^{+}, \tilde{\chi}^{0}\right)}, \\
& d_{e}=d_{e}^{\operatorname{Barr}-\operatorname{Zee}\left(\tilde{\chi}^{+}, \tilde{\chi}^{0}\right)} .
\end{aligned}
$$

Simple estimates are obtained by setting the chargino mass scale $M$ to be much larger than the Higgs mass $100 \mathrm{GeV}[46]$,

$$
\begin{aligned}
& d_{n} \sim\left(\frac{300 \mathrm{GeV}}{M}\right)^{2} \frac{\sin \phi}{\tan \beta} \times 10^{-26} e \mathrm{~cm}, \\
& d_{e} \sim\left(\frac{300 \mathrm{GeV}}{M}\right)^{2} \frac{\sin \phi}{\tan \beta} \times 10^{-27} e \mathrm{~cm} .
\end{aligned}
$$

\footnotetext{
${ }^{4}$ It has recently been shown [48] that similar diagrams mediated by $h$ and $Z$ are also significant for the neutron EDM. This does not affect our numerical estimates.
} 


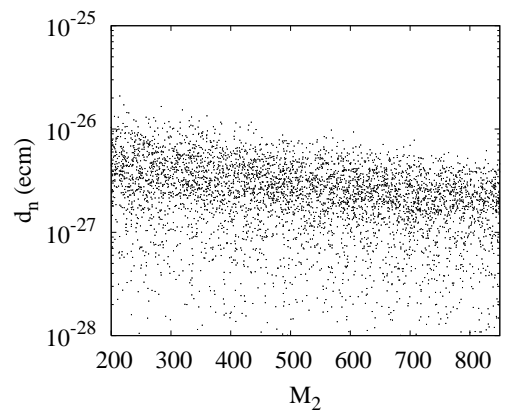

Figure 4: $d_{n}$ in split SUSY. Here $\phi_{\mu}, \phi_{M_{1}}$ are varied in the range $[-\pi, \pi] ; M_{1},|\mu| \in[200$ $\mathrm{GeV}, 1 \mathrm{TeV}]$ and $m_{h} \in[100 \mathrm{GeV}, 300 \mathrm{GeV}]$.

Here $d_{n}$ is obtained by rescaling $d_{e}$ with the factor $m_{q} / m_{e} \sim 10$. The $\tan \beta$ suppression can be traced down to the fact that the EDMs are due to the chargino mass matrix rephasing invariant $M_{11} M_{22} M_{12}^{*} M_{21}^{*} \propto \sin \beta \cos \beta$ and the SM-like Higgs couplings have no $\tan \beta$-enhancement.

We note that since no CEDMs are generated at this level, the mercury EDM is suppressed. The deuteron EDM is, as usual, of order $d_{n}$.

In our numerical analysis, we vary the GUT scale gaugino masses $M_{1,2,3}$ in the range $200 \mathrm{GeV}-1 \mathrm{TeV}$ and $\phi_{\mu}$ in the range $[-\pi, \pi]$. We set the EW scale $\mu^{-}$ parameter by hand in the range $200 \mathrm{GeV}-1 \mathrm{TeV}$ and also vary $m_{h}$ in the range 100 $\mathrm{GeV}-300 \mathrm{GeV}$. Typical $d_{n}$ values are shown in Fig 4 whereas the corresponding $d_{e}$ is found by a simple rescaling. For order one CP phases and low $\tan \beta, d_{n}$ is between $10^{-26}$ and $10^{-27} \mathrm{e} \mathrm{cm}$. Smaller values are obtained at large $\tan \beta$ or for small CP phases/heavy gauginos.

The $d_{n}-d_{e}$ correlations are presented in Fig 12. As expected, $d_{n}$ is almost in one-to-one correspondence with $d_{e}$. The small spread in the EDM values comes from an interplay between the chargino and neutralino Barr-Zee diagrams, which contribute in different proportions to $d_{e}$ and $d_{n}$. In the left figure, the broad tail of $d_{n}$ below $10^{-28} e \mathrm{~cm}$ is an artifact of our numerical procedure and appears due to 1 -loop contributions of heavy but not completely decoupled sfermions (we took $m_{H}, m_{A}=10^{5} \mathrm{GeV}$ and $\left.m_{\text {sferm }}=10^{7} \mathrm{GeV}\right)$.

\section{Conclusions}

We have analyzed correlations between the electron and the neutron EDMs in typical supersymmetric models. Unlike in the case of the $\theta$-term induced electric dipole moments, in SUSY leptonic EDMs are enhanced and are likely to be observed in the next round of experiments.

Assuming that all SUSY CP phases are order one at the GUT scale, lower bounds on the EDMs can be obtained in the decoupling and the split SUSY sce- 
narios:

$$
\begin{array}{ll}
\text { decoupling: } & d_{e} \sim\left(10^{-1}-10^{-2}\right) d_{n} \gtrsim 10^{-28} e \mathrm{~cm}, \\
\text { split SUSY : } & d_{e} \sim 10^{-1} d_{n} \gtrsim 10^{-29} e \mathrm{~cm},
\end{array}
$$

where the lower bound is saturated for a TeV range SUSY spectrum (apart from the first two generation sfermions) in the decoupling case, and at large $\tan \beta$ in the case of split SUSY.

For mSUGRA with small CP phases or a heavy spectrum, no solid lower bound can be derived. However, non-observation of $d_{e}$ at the level $10^{-30} e \mathrm{~cm}$ would imply that either the CP phases are $\lesssim 10^{-5}$ or the scale of SUSY masses is $\gtrsim 100 \mathrm{TeV}$. Both of these options make supersymmetric models very unappealing, so if low energy supersymmetry is indeed realized in nature, one expects $d_{e} \gtrsim 10^{-30} e \mathrm{~cm}$.

Concerning the $d_{n}-d_{e}$ correlations, the main feature of supersymmetry is that it enhances the leptonic EDMs and we observe the following correlations:

$$
\begin{aligned}
\text { small phases : } & d_{e} \sim 10^{-1} d_{n}, \\
\text { heavy spectrum : } & d_{e} \sim 10^{-1} d_{n}, \\
\text { decoupling : } & d_{e} \sim\left(10^{-1}-10^{-2}\right) d_{n}, \\
\text { split SUSY : } & d_{e} \sim 10^{-1} d_{n},
\end{aligned}
$$

where we have assumed that all SUSY CP phases are of the same order of magnitude. These relations as well as correlations with $d_{D}$ and $d_{\mathrm{Hg}}$ can help distinguish supersymmetry from other new physics models.

We note that these correlations are quite stable to variations in the GUT scale soft breaking parameters. As long as $d_{e}$ and $d_{n}$ are generated by similar sets of diagrams, the most important factor for $d_{e} / d_{n}$ is $m_{e} / m_{q}$ and other effects being subleading. 5

In this paper, we have focused on the low and moderate $\tan \beta$ regimes where the effects of four-fermion operators (Fig.(51)) are less important [49] - [51] and have neglected certain two loop RG effects [52]. Also, we have not imposed other experimental constraints such as the bound on the Higgs mass, etc. which are expected to restrict the parameter space further. Clearly, such effects will not change the qualitative picture.

Finally, we note that in the decoupling and the small phases scenarios, at least part of the SUSY spectrum is light and can be observed at the LHC. On the other hand, if the CP phases are order one, the entire SUSY spectrum may lie in the multi-TeV range. Then direct discovery of the superpartners at the LHC may prove to be quite challenging, whereas the EDMs can still probe such a possibility.

\footnotetext{
${ }^{5}$ In particular, breaking the universality between the GUT scale squark and slepton masses does not affect our results significantly: the squark masses at low energies are dictated by the gluino mass and are much less sensitive to $m_{\text {squark }}^{2}(\mathrm{GUT})$.
} 


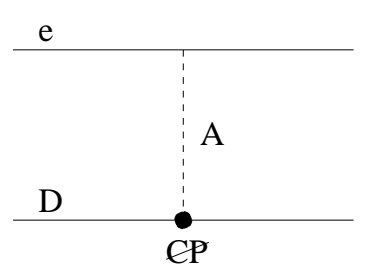

(a)

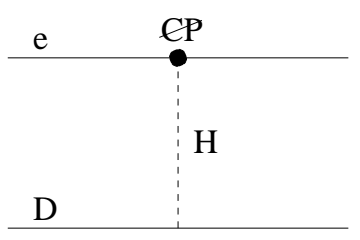

(b)

Figure 5: An example of a four-fermion operator inducing EDMs of composite objects [50]. Here, a pseudoscalar (a) and scalar (b) Higgs exchange induces a CP odd contact interaction $\bar{d} d \bar{e} i \gamma_{5} e$ contributing to $d_{\mathrm{T} 1}$.

Acknowledgements. We are grateful to P. Harris and M. Pospelov for helpful communications. We would also like to thank D. Doyle for collaboration at the early stage of this project. 

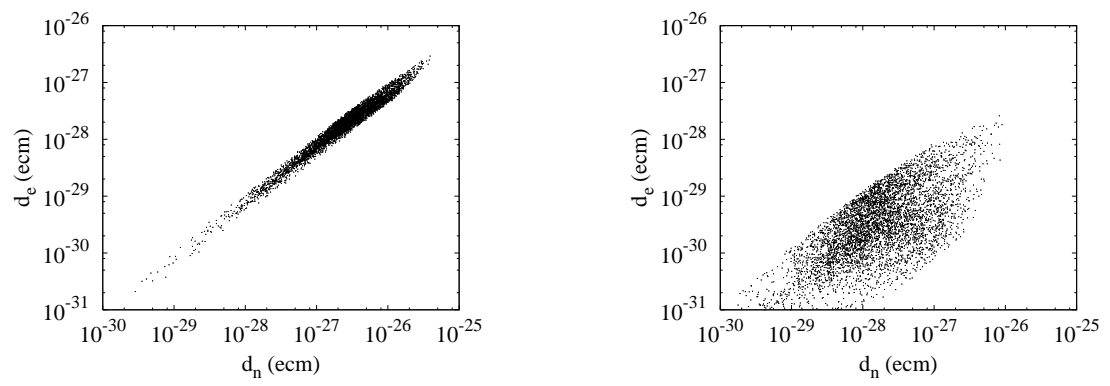

Figure 6: $d_{e}$ vs $d_{n}$ in mSUGRA with small phases, $\tan \beta=5$. Left: $\phi_{\mu} \neq 0$, right: $\phi_{A} \neq 0$.
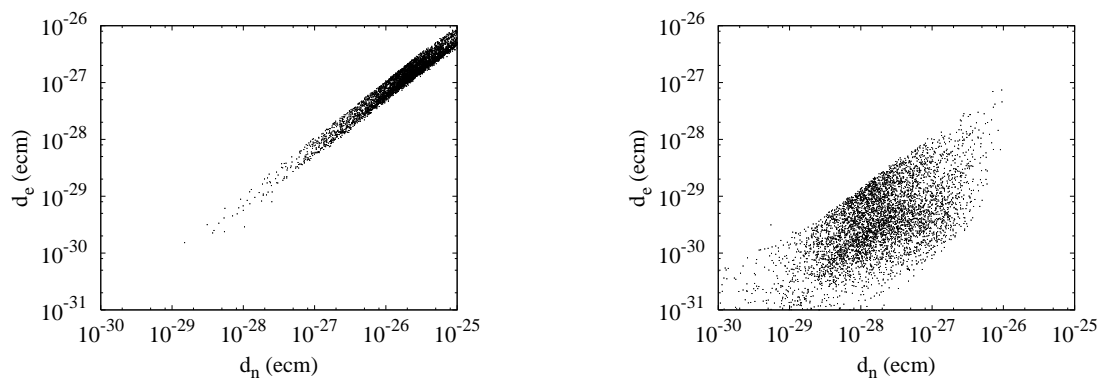

Figure 7: As in Fig [6 but for $\tan \beta=35$.
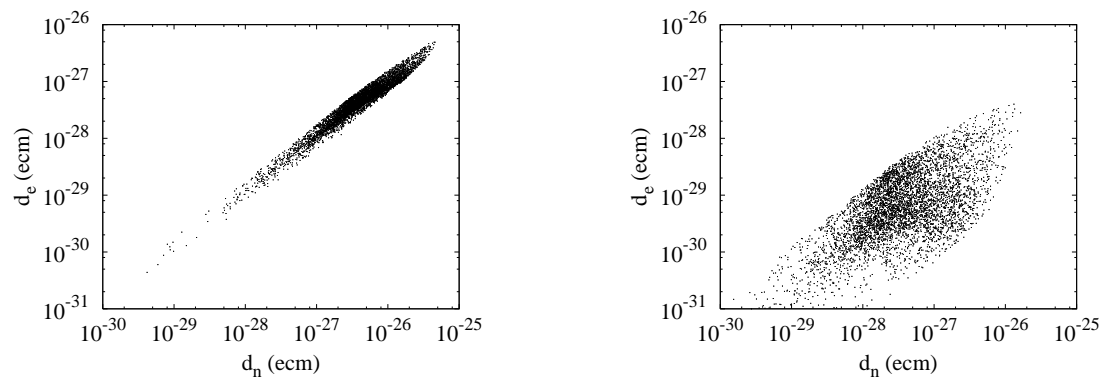

Figure 8: $d_{e}$ vs $d_{n}$ in mSUGRA with a heavy spectrum, $\tan \beta=5$. Left: $\phi_{\mu} \neq 0$, right: $\phi_{A} \neq 0$.
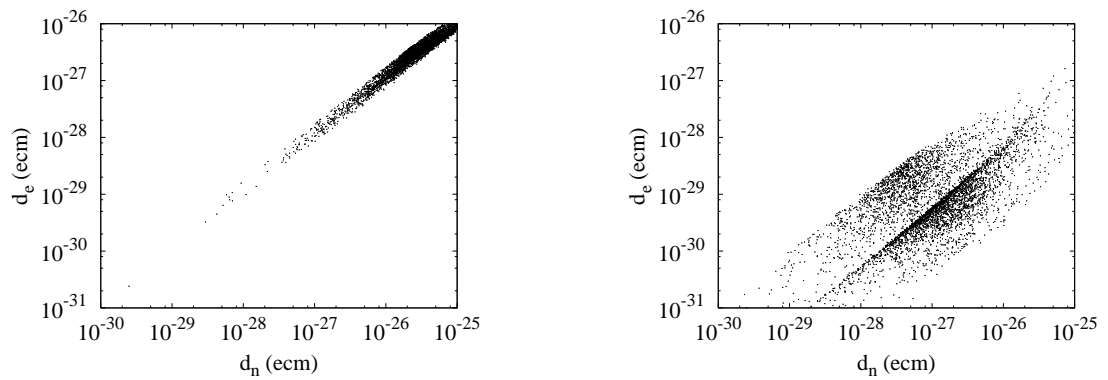

Figure 9: As in Fig 8 but for $\tan \beta=35$. 

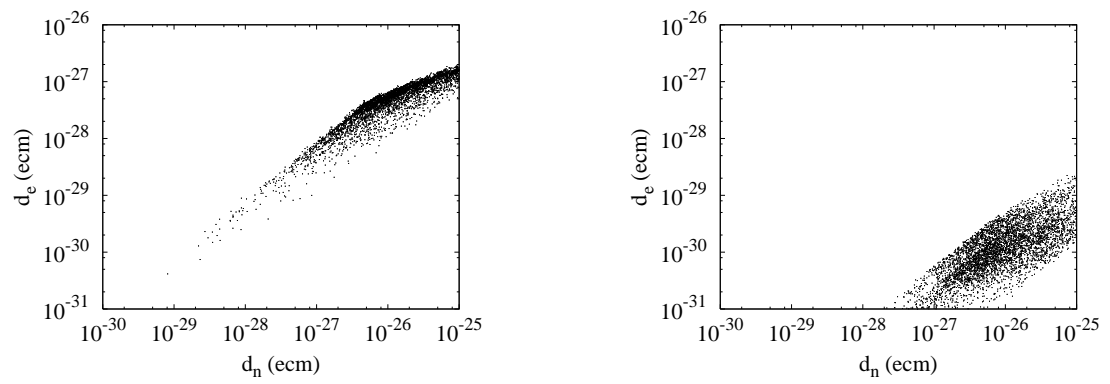

Figure 10: $d_{e}$ vs $d_{n}$ in the decoupling scenario, $\tan \beta=5$. Left: $\phi_{\mu} \neq 0$, right: $\phi_{A} \neq 0$.
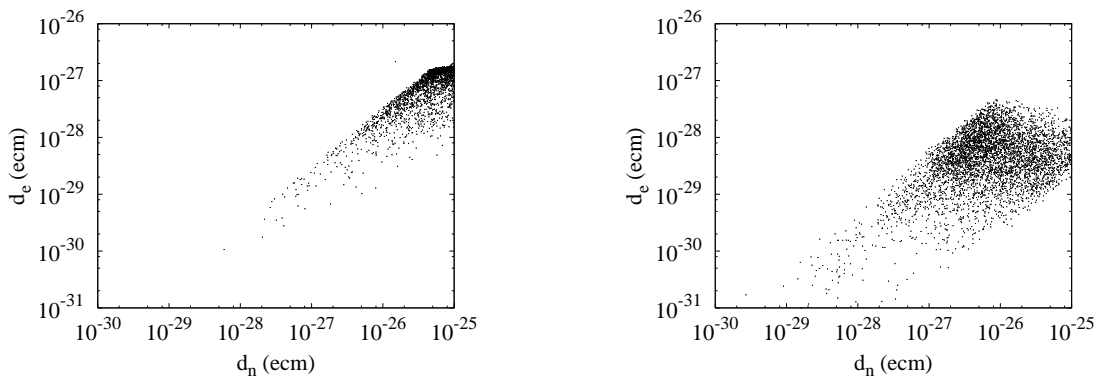

Figure 11: As in Fig 10 but for $\tan \beta=35$.
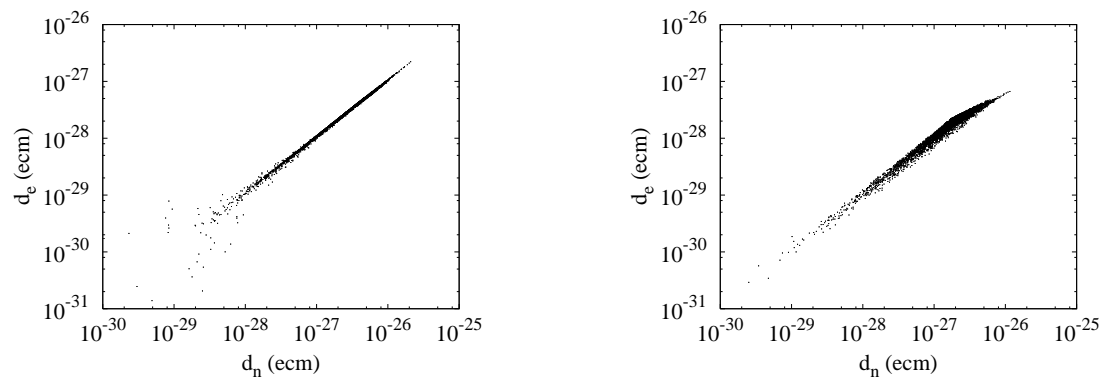

Figure 12: $d_{e}$ vs $d_{n}$ in split SUSY. Left: $\tan \beta=5$, right: $\tan \beta=35$. 


\section{References}

[1] P. G. Harris et al., Phys. Rev. Lett. 82, 904 (1999).

[2] B. C. Regan, E. D. Commins, C. J. Schmidt and D. DeMille, Phys. Rev. Lett. 88, 071805 (2002).

[3] M. V. Romalis, W. C. Griffith and E. N. Fortson, Phys. Rev. Lett. 86, 2505 (2001).

[4] P. Harris, talk at SUSY'05, Durham, 18-23 July 2005, http://susy-2005.dur.ac.uk/susy05programme.html.

[5] D. Kawall, F. Bay, S. Bickman, Y. Jiang and D. DeMille, Phys. Rev. Lett. 92, 133007 (2004).

[6] J. J. Hudson, B. E. Sauer, M. R. Tarbutt and E. A. Hinds, Phys. Rev. Lett. 89, 023003 (2002).

[7] C. Y. Liu and S. K. Lamoreaux, Mod. Phys. Lett. A 19, 1235 (2004); S. K. Lamoreaux, nucl-ex/0109014.

[8] M. van der Grinten, talk at Lepton-Moments, Cape Cod, 9-12 June 2003, http://g2pc1.bu.edu/ leptonmom/program.html, S. Lamoreaux, ibid.

[9] Y. K. Semertzidis et al. [EDM Collaboration], AIP Conf. Proc. 698, 200 (2004), hep-ex/0308063. Y. K. Semertzidis et al., hep-ph/0012087.

[10] J. R. Ellis, S. Ferrara and D. V. Nanopoulos, Phys. Lett. B 114, 231 (1982); W. Buchmuller and D. Wyler, Phys. Lett. B 121, 321 (1983); J. Polchinski and M. B. Wise, Phys. Lett. B 125, 393 (1983).

[11] V. Baluni, Phys. Rev. D 19, 2227 (1979).

[12] R. J. Crewther, P. Di Vecchia, G. Veneziano and E. Witten, Phys. Lett. B 88, 123 (1979).

[13] M. Pospelov and A. Ritz, Phys. Rev. Lett. 83, 2526 (1999); Annals Phys. 318, 119 (2005).

[14] S. Weinberg, Phys. Rev. Lett. 63, 2333 (1989).

[15] R. Arnowitt, J. L. Lopez and D. V. Nanopoulos, Phys. Rev. D 42, 2423 (1990);

R. Arnowitt, M. J. Duff and K. S. Stelle, Phys. Rev. D 43, 3085 (1991).

[16] G. Degrassi, E. Franco, S. Marchetti and L. Silvestrini, JHEP 0511, 044 (2005).

[17] M. Pospelov and A. Ritz, Phys. Rev. D 63, 073015 (2001); D. A. Demir,

M. Pospelov and A. Ritz, Phys. Rev. D 67, 015007 (2003).

[18] J. R. Ellis and R. A. Flores, Phys. Lett. B 377, 83 (1996); J. Hisano and Y. Shimizu, Phys. Rev. D 70, 093001 (2004). 
[19] F. Berruto, T. Blum, K. Orginos and A. Soni, Nucl. Phys. Proc. Suppl. 140, 411 (2005), hep-lat/0411003 E. Shintani et al., hep-lat/0505022.

[20] E. P. Shabalin, Sov. J. Nucl. Phys. 28, 75 (1978) [Yad. Fiz. 28, 151 (1978)]; M. B. Gavela, A. Le Yaouanc, L. Oliver, O. Pene, J. C. Raynal and T. N. Pham, Phys. Lett. B 109, 215 (1982); I. B. Khriplovich and A. R. Zhitnitsky, Phys. Lett. B 109, 490 (1982).

[21] R. D. Peccei and H. R. Quinn, Phys. Rev. Lett. 38, 1440 (1977).

[22] S. M. Barr and D. Seckel, Phys. Rev. D 46, 539 (1992); R. Holman et al., Phys. Lett. B 282, 132 (1992).

[23] R. N. Mohapatra and G. Senjanovic, Phys. Lett. B 79, 283 (1978); S. M. Barr, Phys. Rev. Lett. 53, 329 (1984); A. E. Nelson, Phys. Lett. B 136, 387 (1984).

[24] O. Lebedev, K. A. Olive, M. Pospelov and A. Ritz, Phys. Rev. D 70, 016003 (2004).

[25] V. F. Dmitriev and R. A. Sen'kov, Phys. Atom. Nucl. 66, 1940 (2003); J. S. M. Ginges and V. V. Flambaum, Phys. Rept. 397, 63 (2004).

[26] I. B. Khriplovich and S. K. Lamoreaux, "CP Violation Without Strangeness", Springer, 1997.

[27] J. P. Archambault, A. Czarnecki and M. Pospelov, Phys. Rev. D 70, 073006 (2004); M. E. Pospelov and I. B. Khriplovich, Sov. J. Nucl. Phys. 53, 638 (1991) [Yad. Fiz. 53, 1030 (1991)].

[28] S. Abel, S. Khalil and O. Lebedev, Phys. Rev. Lett. 89, 121601 (2002).

[29] K. S. Babu, B. Dutta and R. N. Mohapatra, Phys. Rev. D 65, 016005 (2002).

[30] S. Abel, D. Bailin, S. Khalil and O. Lebedev, Phys. Lett. B 504, 241 (2001); J. L. Diaz-Cruz and J. Ferrandis, hep-ph/0504094.

[31] T. Falk and K. A. Olive, Phys. Lett. B 375, 196 (1996); T. Ibrahim and P. Nath, Phys. Rev. D 57, 478 (1998); Phys. Rev. D 58, 111301 (1998); M. Brhlik, G. J. Good and G. L. Kane, Phys. Rev. D 59, 115004 (1999).

[32] T. Falk, K. A. Olive, M. Pospelov and R. Roiban, Nucl. Phys. B 560, 3 (1999).

[33] V. D. Barger, T. Falk, T. Han, J. Jiang, T. Li and T. Plehn, Phys. Rev. D 64, 056007 (2001).

[34] S. Abel, S. Khalil and O. Lebedev, Nucl. Phys. B 606, 151 (2001).

[35] S. Abel, S. Khalil and O. Lebedev, Phys. Rev. Lett. 86, 5850 (2001).

[36] V. S. Kaplunovsky and J. Louis, Phys. Lett. B 306, 269 (1993); A. Brignole, L. E. Ibanez and C. Munoz, hep-ph/9707209. See also L. E. Ibanez, Phys. Rev. D 71, 055005 (2005).

[37] O. Lebedev and S. Morris, JHEP 0208, 007 (2002). 
[38] A. Falkowski, O. Lebedev and Y. Mambrini, hep-ph/0507110.

[39] P. Nath, Phys. Rev. Lett. 66, 2565 (1991); Y. Kizukuri and N. Oshimo, Phys. Rev. D 45, 1806 (1992).

[40] J. Bagger, J. L. Feng and N. Polonsky, Nucl. Phys. B 563, 3 (1999).

[41] J. Dai, H. Dykstra, R. G. Leigh, S. Paban and D. Dicus, Phys. Lett. B 237, 216 (1990) [E-ibid. B 242, 547 (1990)].

[42] S. M. Barr and A. Zee, Phys. Rev. Lett. 65, 21 (1990).

[43] D. Chang, W. Y. Keung and A. Pilaftsis, Phys. Rev. Lett. 82, 900 (1999).

[44] D. Chang, W. F. Chang and W. Y. Keung, Phys. Rev. D 66, 116008 (2002); A. Pilaftsis, Nucl. Phys. B 644, 263 (2002).

[45] N. Arkani-Hamed and S. Dimopoulos, hep-th/0405159.

[46] N. Arkani-Hamed, S. Dimopoulos, G. F. Giudice and A. Romanino, Nucl. Phys. B 709, 3 (2005).

[47] D. Chang, W. F. Chang and W. Y. Keung, Phys. Rev. D 71, 076006 (2005).

[48] G. F. Giudice and A. Romanino, "Electric dipole moments in split supersymmetry," hep-ph/0510197.

[49] S. M. Barr, Phys. Rev. Lett. 68, 1822 (1992).

[50] O. Lebedev and M. Pospelov, Phys. Rev. Lett. 89, 101801 (2002).

[51] D. A. Demir, O. Lebedev, K. A. Olive, M. Pospelov and A. Ritz, Nucl. Phys. B 680, 339 (2004).

[52] K. A. Olive, M. Pospelov, A. Ritz and Y. Santoso, hep-ph/0506106. 